\title{
A COHOMOLOGICAL OBSTRUCTION TO WEAK APPROXIMATION FOR HOMOGENEOUS SPACES
}

\author{
MIKHAIL BOROVOI AND TOMER M. SCHLANK
}

\begin{abstract}
Let $X$ be a homogeneous space, $X=G / H$, where $G$ is a connected linear algebraic group over a number field $k$, and $H \subset G$ is a $k$-subgroup (not necessarily connected). Let $S$ be a finite set of places of $k$. We compute a Brauer-Manin obstruction to weak approximation for $X$ in $S$ in terms of Galois cohomology.
\end{abstract}

\section{CONTEnTs}

0. Introduction

1. Preliminaries on the Brauer group and the Brauer-Manin obstruction

2. Preliminaries on quasi-trivial groups

3. The cohomological obstruction $c_{S}$

4. Proofs: the case of a torus

5. Proofs: the general case

References

\section{INTRODUCTION}

Let $X$ be an algebraic variety over a number field $k$, and let $S$ be a finite set of places of $k$. We say that $X$ has the weak approximation property in $S$ if $X(k)$ is dense in $\prod_{v \in S} X\left(k_{v}\right)$ with respect to the diagonal embedding. We say that $X$ has the weak approximation property if it has the weak approximation property in $S$ for any finite set $S$ of places of $k$.

In 1970 Manin [M] introduced a general obstruction to the Hasse principle for a $k$-variety $X$, using the Brauer group of $X$. Using Manin's ideas, Colliot-Thélène and Sansuc [CTS] defined a Brauer-Manin obstruction to weak approximation for $X$ in all $S$ simultaneously (see also [Sk], §5.2). We consider a variation of this obstruction introduced in [B1], which is a Brauer-Manin obstruction to weak approximation in a specific set of places $S$ for a $k$-variety $X$ having a $k$-point.

Write $k_{S}=\prod_{v \in S} k_{v}$, then

$$
X\left(k_{S}\right)=\prod_{v \in S} X\left(k_{v}\right) .
$$

We assume that $X(k) \neq \emptyset$. The Brauer-Manin obstruction of [B1] is a map

$$
\mathrm{ob}_{S}: X\left(k_{S}\right) \rightarrow \mathrm{Б}_{S, \emptyset}(X)^{D},
$$

2000 Mathematics Subject Classification. Primary: 14M17; Secondary: 14G05, 20G10, 20 G30.

Key words and phrases. Brauer-Manin obstruction, weak approximation, homogeneous spaces, linear algebraic groups, Brauer group, Galois cohomology.

This research was partially supported by the Hermann Minkowski Center for Geometry and by ISF grant $807 / 07$. 
where $\mathrm{Б}_{S, \emptyset}(X):=\mathrm{Б}_{S}(X) / \mathrm{Б}(X)$ is a certain subquotient of the Brauer group $\operatorname{Br} X$ (for details see $\$ 11)$ and ${ }^{D}$ denotes the dual group, i.e. $\bullet^{D}=\operatorname{Hom}(\bullet, \mathbb{Q} / \mathbb{Z})$. The $\operatorname{map} \operatorname{ob}_{S}$ is an obstruction in the following sense: if $x_{S} \in X\left(k_{S}\right)$ and $\operatorname{ob}_{S}\left(x_{S}\right) \neq 0$, then $x_{S}$ is not contained in the closure of $X(k)$ in $X\left(k_{S}\right)$. In particular, if the map obs is not identically 0 , then $X$ does not have weak approximation in $S$.

Now let $X$ be a homogeneous space of a connected linear $k$-group $G$. It is convenient to use the notion of a quasi-trivial group, introduced by Colliot-Thelene, see Definition 2.1 in [CT] or Definition 2.2 below. By Lemma 2.3 below we may assume that $X$ is a homogeneous space of a quasi-trivial $k$-group $G$. In [B2] we computed the Brauer-Manin obstruction of [B1] to the Hasse principle for $X$ in terms of Galois cohomology. Here we do a similar computation for the Brauer-Manin obstruction of [B1] to weak approximation for $X$. We assume that $X$ has a $k$-point $x^{0}$. Let $H$ denote the stabilizer of $x^{0}$ in $G$, then $X=G / H$.

From now on we assume that $X=G / H$, where $G$ is quasi-trivial (and $H$ is not necessarily connected). We describe the group $\mathrm{b}_{S, \emptyset}(X)^{D}$ and the map $\mathrm{ob}_{S}$ in terms of Galois cohomology. Let $H^{\text {mult }}$ denote the greatest quotient of $H$ that is a group of multiplicative type. Write

$$
H^{1}\left(k_{S}, H^{\text {mult }}\right)=\prod_{v \in S} H^{1}\left(k_{v}, H^{\text {mult }}\right)
$$

and set

$$
\mathrm{บ}_{S}^{1}\left(k, H^{\text {mult }}\right)=\operatorname{coker}\left[H^{1}\left(k, H^{\text {mult }}\right) \stackrel{\operatorname{loc}_{S}}{\longrightarrow} H^{1}\left(k_{S}, H^{\text {mult }}\right)\right],
$$

where $\operatorname{loc}_{S}$ is the localization map. The following theorem describes the group $\mathrm{Б}_{S, \emptyset}(X)^{D}$ in terms of Galois cohomology.

Theorem 0.1 (Theorem 5.1). Let $X=G / H$, where $G$ is a quasi-trivial k-group over a number field $k$, and $H$ is a $k$-subgroup of $G$. Then there is a canonical isomorphism:

$$
\phi: \mathrm{Б}_{S, \emptyset}(X)^{D} \stackrel{\sim}{\rightarrow} \mathrm{\Psi}_{S}^{1}\left(k, H^{\text {mult }}\right) .
$$

We wish to describe not only the group $\mathrm{Б}_{S, \emptyset}(X)^{D}$, but also the map ob $\mathrm{ob}_{S}$ in terms of Galois cohomology. Let $x_{S}=\left(x_{v}\right)_{v \in S} \in X\left(k_{S}\right)$. For $v \in S$ consider the $G\left(k_{v}\right)$-orbit $G\left(k_{v}\right) \cdot x_{v}$ of $x_{v}$ in $X\left(k_{v}\right)$. This orbit defines a cohomology class

$$
\xi_{v}\left(x_{v}\right) \in \operatorname{ker}\left[H^{1}\left(k_{v}, H\right) \rightarrow H^{1}\left(k_{v}, G\right)\right],
$$

cf. [Se, §I.5.4]. Consider the canonical epimorphism $\mu: H \rightarrow H^{\text {mult }}$, and set

$$
\begin{aligned}
& \xi_{v}^{\text {mult }}\left(x_{v}\right)=\mu_{*}\left(\xi_{v}\left(x_{v}\right)\right) \in H^{1}\left(k_{v}, H^{\text {mult }}\right), \\
& \xi_{S}^{\text {mult }}\left(x_{S}\right)=\left(\xi_{v}^{\text {mult }}\left(x_{v}\right)\right)_{v \in S} \in H^{1}\left(k_{S}, H^{\text {mult }}\right) .
\end{aligned}
$$

Let $c_{S}\left(x_{S}\right)$ denote the image of $\xi_{S}^{\text {mult }}\left(x_{S}\right)$ under the canonical map

$$
H^{1}\left(k_{S}, H^{\text {mult }}\right) \rightarrow \mathrm{บ}_{S}^{1}\left(k, H^{\text {mult }}\right)
$$

The following theorem describes the map $\mathrm{ob}_{S}$ in terms of Galois cohomology.

Theorem 0.2 (Theorem [5.1). Let k, G, H, and $X$ be as in Theorem 0.1. The following diagram commutes:

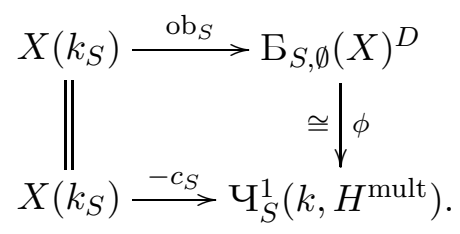


Note that results similar to our Theorem 0.2 in the special case of a finite group $H$ were obtained earlier by D. Harari [H], §5, Theorem 4, and C. Demarche [D1], §7, Corollary 4.

Remark 0.3. In this paper we consider the Brauer-Manin obstruction ob ${ }_{S}$ of [B1, which is a variation of the Brauer-Manin obstruction associated with the group $\mathrm{B}_{\omega}(X)$, cf. [Sa, (6.2.3)]. By [B1, Thm. 2.4], if $H$ is connected or abelian, then $\mathrm{ob}_{S}$ is the only obstruction to weak approximation in $S$ for $X$. However, it may be not the only obstruction if $H$ is non-connected and non-abelian, see Demarche [D1, §6, Prop. 2]. The failure of weak approximation in the counter-example of Demarche can be explained by the Brauer-Manin obstruction associated with the larger group $\mathrm{Br}_{\mathrm{nr}, 1}(X)$, loc. cit.

Now let $H$ be an arbitrary $k$-group of multiplicative type. Let $\widehat{H}:=\operatorname{Hom}_{\bar{k}}\left(H_{\bar{k}}, \mathbb{G}_{m, \bar{k}}\right)$ be the (geometric) character group of $H$. Let $\amalg_{S}^{1}(k, \widehat{H})$ denote the kernel of the localization map

$$
\operatorname{loc}_{S^{\complement}}^{1}=\operatorname{loc}_{\mathscr{V}(k) \backslash S}^{1}: H^{1}(k, \widehat{H}) \rightarrow \prod_{v \in \mathscr{V}(k) \backslash S} H^{1}\left(k_{v}, \widehat{H}\right)
$$

where $\mathscr{V}(k)$ is the set of all places of $k$. As a byproduct of our study of the group $\mathrm{บ}^{1}\left(k, H^{\text {mult }}\right)$ we obtain a duality theorem:

Theorem 0.4 (Theorem 4.2). Let $H$ be a k-group of multiplicative type over a number field $k$. There is a canonical non-degenerate pairing

$$
\amalg_{S}^{1}(k, \widehat{H}) / \amalg_{\emptyset}^{1}(k, \widehat{H}) \times \Psi_{S}^{1}(k, H) \stackrel{\cup_{S}}{\longrightarrow} \mathbb{Q} / \mathbb{Z} .
$$

Theorem 0.4 generalizes a result of Sansuc [Sa], Lemma 1.4, who considered the case of finite $H$. This theorem can be also deduced from the Poitou-Tate exact sequence for groups of multiplicative type, see [D2, Thm. 6.3].

Acknowledgements. The authors are grateful to David Harari for useful discussions and to the referee for helpful comments. The first-named author worked on the paper while visiting the Max-Planck-Institut für Mathematik, Bonn; he thanks the Institute for hospitality, support, and excellent working conditions.

\section{Notation.}

By $k$ we denote a field of characteristic 0 , and by $\bar{k}$ a fixed algebraic closure of $k$. By a $k$-variety we mean a separated geometrically integral scheme of finite type over $k$. For a $k$-variety $X$ we set $\bar{X}=X \times_{k} \bar{k}$ and

$$
U(\bar{X})=\bar{k}[X]^{*} / \bar{k}^{*},
$$

where $\bar{k}[X]$ is the ring of regular functions on $\bar{X}$ and $\bar{k}[X]^{*}$ is the group of invertible regular functions on $\bar{X}$. We denote by $\operatorname{Pic} \bar{X}$ the Picard group of $\bar{X}$. Note that both $U(\bar{X})$ and $\operatorname{Pic} \bar{X}$ are Galois modules, i.e. the Galois group $\operatorname{Gal}(\bar{k} / k)$ acts on them.

By an algebraic $k$-group $H$ we mean a linear algebraic group (not necessarily connected). We write $\widehat{H}$ or $\mathbb{X}(H)$ for the (geometric) character group of $H$, i.e.

$$
\mathbb{X}(H)=\widehat{H}:=\operatorname{Hom}_{\bar{k}}\left(\bar{H}, \mathbb{G}_{m, \bar{k}}\right) .
$$

When $k$ is assumed to be a number field, we write $\mathscr{V}(k)$ for the set of places of $k$. If $v \in \mathscr{V}(k)$, we write $k_{v}$ for the completion of $k$ at $v$. Let $S \subset \mathscr{V}(k)$ be a finite set of places of $k$. We set $k_{S}=\prod_{v \in S} k_{v}$, then for a $k$-variety $X$ we have

$$
X\left(k_{S}\right)=\prod_{v \in S} X\left(k_{v}\right)
$$


The set of $k$-points $X(k)$ embeds diagonally into $X\left(k_{S}\right)$, and we denote by $\overline{X(k)}$ the closure of $X(k)$ in $X\left(k_{S}\right)$. If $G$ is a linear algebraic group over $k$, we set

$$
H^{1}\left(k_{S}, G\right):=\prod_{v \in S} H^{1}\left(k_{v}, G\right)
$$

\section{Preliminaries on the Brauer group and the Brauer-Manin obstruction}

Let $k$ be a field of characteristic 0 . Let $X$ be a smooth geometrically integral $k$-variety with a marked $k$-point $x^{0}$. Then $\operatorname{Br} X:=H_{\text {ét }}^{2}\left(X, \mathbb{G}_{m}\right)$ is the cohomological Brauer group of $X$. We use the following notation:

$$
\begin{aligned}
\operatorname{Br}_{0} X & =\operatorname{im}[\operatorname{Br} k \rightarrow \operatorname{Br} X] ; \quad \operatorname{Br}_{1} X=\operatorname{ker}\left[\operatorname{Br} X \rightarrow \operatorname{Br} X_{\bar{k}}\right] ; \\
\operatorname{Br}_{x^{0}} X & =\operatorname{ker}\left[\left(x^{0}\right)^{*}: \operatorname{Br}_{1} X \rightarrow \operatorname{Br} k\right] ; \quad \operatorname{Br}_{\mathrm{a}} X=\operatorname{Br}_{1} X / \operatorname{Br}_{0} X .
\end{aligned}
$$

We have

$$
\mathrm{Br}_{1} X=\mathrm{Br}_{0} X \oplus \mathrm{Br}_{x^{0}} X
$$

and therefore we have a canonical isomorphism $\mathrm{Br}_{x^{0}} X \stackrel{\sim}{\rightarrow} \mathrm{Br}_{\mathrm{a}} X$.

If $S \subset \mathscr{V}(k)$ is a finite subset, let $\mathrm{Б}_{S}(X)$ be the subgroup of $\operatorname{Br}_{x^{0}} X$ consisting of elements $b$ whose localizations $\operatorname{loc}_{v} b$ in $\operatorname{Br} X_{k_{v}}$ are trivial for all places $v$ of $k$ outside $S$. We set $\mathrm{Б}(X)=\mathrm{Б}_{\emptyset}(X)$.

Note that for any $S$ we have $\mathrm{Б}_{\emptyset}(X) \subset \mathrm{Б}_{S}(X)$. We denote

$$
\mathrm{Б}_{S, \emptyset}(X):=\mathrm{Б}_{S}(X) / \mathrm{Б}_{\emptyset}(X)=\mathrm{Б}_{S}(X) / \mathrm{Б}(X) .
$$

Now we describe the Brauer-Manin obstruction of [B1] to weak approximation in $S$ for $X$.

For our purposes the Brauer-Manin obstruction coming from the subgroup $\operatorname{Br}_{1} X$ of $\operatorname{Br} X$ will suffice. Following [Sa] and [B1], we define the Brauer-Manin obstruction in terms of the group $\mathrm{5}_{S, \emptyset}(X)$.

Let $X$ be a smooth geometrically integral variety over a field $k$ of characteristic 0 . Consider the pairing

$$
X(k) \times \mathrm{Br}_{1} X \stackrel{\mathrm{ev}}{\rightarrow} \mathrm{Br} k
$$

where ev is the evaluation map ev: $(x, b) \mapsto b(x)$. This pairing is additive in $b$ :

$$
\mathrm{ev}\left(x, b+b^{\prime}\right)=\mathrm{ev}(x, b)+\mathrm{ev}\left(x, b^{\prime}\right) .
$$

If $k$ is a local field, then the above pairing gives us a pairing

$$
\begin{gathered}
X(k) \times \operatorname{Br}_{1} X \stackrel{\text { invoev }}{\longrightarrow} \mathbb{Q} / \mathbb{Z}, \\
(x, b) \mapsto \operatorname{inv}(b(x))
\end{gathered}
$$

where inv: $\operatorname{Br} k \rightarrow \mathbb{Q} / \mathbb{Z}$ is the homomorphism of local class field theory. This pairing is continuous in $x$ (see [Sa], Lemma 6.2, or [BD], Lemma 6.2) and is additive in $b$.

Now let $k$ be a number field. Let $S \subset \mathscr{V}(k)$ be a finite subset. Consider the pairing

$$
\begin{gathered}
\langle,\rangle_{S}: X\left(k_{S}\right) \times \mathrm{Б}_{S}(X) \rightarrow \mathbb{Q} / \mathbb{Z}, \\
\left\langle\left(x_{v}\right)_{v \in S}, b\right\rangle_{S}=\sum_{v \in S}\left(\operatorname{inv}_{v}\left(b\left(x_{v}\right)\right)\right) .
\end{gathered}
$$

If $b \in \mathrm{5}_{\emptyset}(X)$, then $\left\langle x_{S}, b\right\rangle_{S}=0$ for any $x_{S} \in X\left(k_{S}\right)$. Thus the pairing $\langle,\rangle_{S}$ induces a pairing

$$
X\left(k_{S}\right) \times \mathrm{Б}_{S}(X) / \mathrm{Б}_{\emptyset}(X) \rightarrow \mathbb{Q} / \mathbb{Z}
$$


which is additive in the second argument. We call the pairings (11) and (2) the Manin pairings. Thus we obtain a map

$$
\mathrm{ob}_{S}: X\left(k_{S}\right) \rightarrow\left(\mathrm{Б}_{S}(X) / \mathrm{Б}_{\emptyset}(X)\right)^{D}=\mathrm{Б}_{S, \emptyset}(X)^{D} .
$$

The map obs is continuous because the pairing $\langle,\rangle_{S}$ is continuous in $x_{S}$. Further, by the Hasse-Brauer-Noether theorem if $x \in X(k) \subset X\left(k_{S}\right)$, then $\operatorname{ob}_{S}(x)=0$. It follows that if $x_{S}$ is contained in the closure $\overline{X(k)}$ of $X(k)$ in $X\left(k_{S}\right)$, then ob $\operatorname{ob}_{S}\left(x_{S}\right)=0$.

Recall that $X$ has the weak approximation property in $S$, if $X(k)$ is dense in $X\left(k_{S}\right)$. If $X$ has the weak approximation property in $S$, then $\overline{X(k)}=X\left(k_{S}\right)$ and $\mathrm{ob}_{S}$ is identically 0 . We see therefore that $\mathrm{ob}_{S}$ is an obstruction to weak approximation in $S$. We call ob the Brauer-Manin obstruction to weak approximation in $S$, associated with $\mathrm{5}_{S, \emptyset}$.

The obstruction $\mathrm{ob}_{S}$ is functorial. Namely, let $\pi:\left(X, x^{0}\right) \rightarrow\left(Y, y^{0}\right)$ be a morphism of $k$-varieties with marked $k$-points. Then the following diagram is commutative:

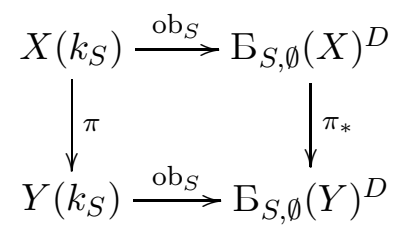

where $\pi_{*}$ is the homomorphism induced by $\pi$.

Remark 1.1. In [B1] the group $\mathrm{b}_{S}(X)$ and the Brauer-Manin obstruction were defined in terms of $\mathrm{Br}_{\mathrm{a}} X=\mathrm{Br}_{1} X / \mathrm{Br}_{0} X$ rather than $\mathrm{Br}_{x^{0}} X$. However, these two groups are canonically isomorphic, and one can check that our definitions here are essentially equivalent to those of [B1]. Note that though we defined the obstruction ob $b_{S}$ using $x^{0}$, this obstruction essentially does not depend on $x^{0}$, cf. [B1], $\S 1$.

\section{Preliminaries on Quasi-trivial groups}

All the lemmas in this section are well known. For the reader's convenience we provide short proofs and/or references.

2.1. Notation concerning linear algebraic groups. Let $G$ be a connected linear algebraic group over a field $k$ of characteristic 0 . We use the following notation:

$G^{\mathrm{u}}$ is the unipotent radical of $G$;

$G^{\mathrm{red}}=G / G^{\mathrm{u}}$, it is a reductive $k$-group;

$G^{\text {ss }}$ is the commutator subgroup of $G^{\text {red }}$, it is a semisimple $k$-group; $G^{\text {tor }}=G^{\text {red }} / G^{\text {ss }}$, it is a $k$-torus.

Let $H$ be a linear $k$-group, not necessarily connected. We denote by $H^{\text {mult }}$ the biggest quotient group of $H$ that is a group of multiplicative type. Let $\widehat{H}$ denote the character group of $H$. We have $\widehat{H}=\widehat{H^{\text {mult }}}$. If $H$ is connected, then $H^{\text {mult }}=H^{\text {tor }}$.

Recall that a torus $T$ is called quasi-trivial if its character group $\widehat{T}$ is a permutation Galois module.

Definition 2.2 ( $[\mathrm{CT}$, Proposition 2.2). Let $k$ be a field and $G$ a connected linear $k$-group. We say that $G$ is quasi-trivial if $G^{\text {tor }}$ is quasi-trivial and $G^{\text {ss }}$ is simply connected.

Let $X$ be a homogeneous space of a connected linear $k$-group $G$. By virtue of the following lemma one can always take $G$ to be quasi-trivial without changing $X$. 
Lemma 2.3. Let $G$ be a connected linear algebraic group over a field $k$ of characteristic 0 , then there exists a surjective homomorphism $G^{\prime} \rightarrow G$ such that $G^{\prime}$ is a quasi-trivial $k$-group.

Proof. See [CT], Proposition-Definition 3.1.

2.4. By a pair of $k$-groups we mean a pair $(G, H)$, where $G$ is a quasi-trivial $k$-group and $H$ is a $k$-subgroup of $G$ (not necessarily connected). A pair $(G, H)$ defines a homogeneous space $X:=G / H$ together with a marked point $x^{0}=e H \in X(k)$, where $e \in G(k)$ is the identity element of $G$.

By a morphism of pairs $\phi:\left(G_{1}, H_{1}\right) \rightarrow\left(G_{2}, H_{2}\right)$ we mean a surjective homomorphism $\phi: G_{1} \rightarrow G_{2}$ such that $\phi\left(H_{1}\right)=H_{2}$. If we set $X_{1}=G_{1} / H_{1}$ and $X_{2}=G_{2} / H_{2}$, then we have an induced morphism $\phi_{*}:\left(X_{1}, x_{1}^{0}\right) \rightarrow\left(X_{2}, x_{2}^{0}\right)$, where $x_{1}^{0}$ and $x_{2}^{0}$ are the corresponding marked points.

Let $G$ be a $k$-group. In the next lemma we use the notation $\mathbb{X}(G)=\widehat{G}$ to denote the Galois module of (geometric) characters of $G$.

Proposition 2.5. Let $(G, H)$ be a pair of k-groups with quasi-trivial $G$ as in 2.4, and let $X=G / H$. Let $\bar{X}=X \times_{k} \bar{k}$. Consider the natural morphism of Galois modules

$$
\mathbb{X}(G) \rightarrow \mathbb{X}(H)
$$

and the dual morphism of $k$-groups of multiplicative type

$$
H^{\text {mult }} \rightarrow G^{\text {mult }}=G^{\text {tor }}
$$

Then there are natural isomorphisms of Galois modules

(i) $U(\bar{X}) \cong \operatorname{ker}[\mathbb{X}(G) \rightarrow \mathbb{X}(H)] \cong \mathbb{X}\left(\operatorname{coker}\left[H^{\text {mult }} \rightarrow G^{\text {tor }}\right]\right)$

(ii) $\operatorname{Pic} \bar{X} \cong \operatorname{coker}[\mathbb{X}(G) \rightarrow \mathbb{X}(H)] \cong \mathbb{X}\left(\operatorname{ker}\left[H^{\text {mult }} \rightarrow G^{\text {tor }}\right]\right)$

Proof. We have canonical isomorphisms

$$
\operatorname{ker}[\mathbb{X}(G) \rightarrow \mathbb{X}(H)] \cong \mathbb{X}\left(\operatorname{coker}\left[H^{\text {mult }} \rightarrow G^{\text {tor }}\right]\right)
$$

and

$$
\operatorname{coker}[\mathbb{X}(G) \rightarrow \mathbb{X}(H)] \cong \mathbb{X}\left(\operatorname{ker}\left[H^{\text {mult }} \rightarrow G^{\mathrm{tor}}\right]\right)
$$

Proof of (ii). The natural map $G \rightarrow G / H=X$ induces an embedding $U(\bar{X}) \hookrightarrow$ $U(\bar{G})$. By Rosenlicht's theorem ( $[\mathbb{R}]$, Theorem 3) the injection $\mathbb{X}(G) \hookrightarrow \bar{k}[\bar{G}]^{*}$ induces an isomorphism $\mathbb{X}(G) \cong U(\bar{G})$. It is easy to see that a character $\chi \in \mathbb{X}(G)$ corresponds to an element of $U(\bar{G})$ coming from $U(\bar{X})$ if and only if $\chi \in \operatorname{ker}[\mathbb{X}(G) \rightarrow \mathbb{X}(H)]$.

Proof of (iii). See Popov $[\mathrm{P}]$, Theorem 4 (we use that Pic $\bar{G}=0$, cf. [CT], Definition 2.1).

\section{The Cohomological obstruction $c_{S}$}

In this section, using nonabelian Galois cohomology we define a cohomological obstruction to weak approximation in $X$ at a finite set of places $S \subset \mathscr{V}(k)$. This obstruction takes values in the group $\mathrm{\Psi}_{S}^{1}\left(k, H^{\text {mult }}\right)$, and we denote it by $c_{S}$.

Let $k$ be a number field. Let $\bar{k}$ be a fixed algebraic closure of $k$. Let $(G, H)$ be a pair of $k$-groups as in 2.4 over a number field $k$.

Lemma 3.1. Let $S$ be a finite subset of $\mathscr{V}(k),(G, H)$ be a pair of $k$-groups as in 2.4. and $x_{S} \in X\left(k_{S}\right)$ be a $k_{S}$-point. Then $x_{S} \in \overline{X(k)}$ if and only if the orbit $G\left(k_{S}\right) \cdot x_{S}$ of $x_{S}$ contains a k-point of $X$. 
Proof. This is well known, see e.g. [B3], §2.1.

We see from Lemma 3.1 that a point $x_{S} \in X\left(k_{S}\right)$ lies in the closure $\overline{X(k)}$ of $X(k)$ if and only if its $G\left(k_{S}\right)$-orbit $G\left(k_{S}\right) \cdot x_{S}$ lies in in the image of the localization map

$$
\operatorname{loc}_{S}: G(k) \backslash X(k) \rightarrow G\left(k_{S}\right) \backslash X\left(k_{S}\right) .
$$

3.2. Cohomological formulation. By [Se, I.5.5, Corollary 1 of Proposition 36, we have a canonical bijection

$$
\tau_{k}: G(k) \backslash X(k) \stackrel{\sim}{\longrightarrow} \operatorname{ker}\left[H^{1}(k, H) \rightarrow H^{1}(k, G)\right] .
$$

For a finite set $S$ of places of $k$ we obtain a bijection

$$
\tau_{S}=\prod_{v \in S} \tau_{k_{v}}: G\left(k_{S}\right) \backslash X\left(k_{S}\right) \rightarrow \operatorname{ker}\left[H^{1}\left(k_{S}, H\right) \rightarrow H^{1}\left(k_{S}, G\right)\right]
$$

We have a commutative diagram with bijective vertical arrows:

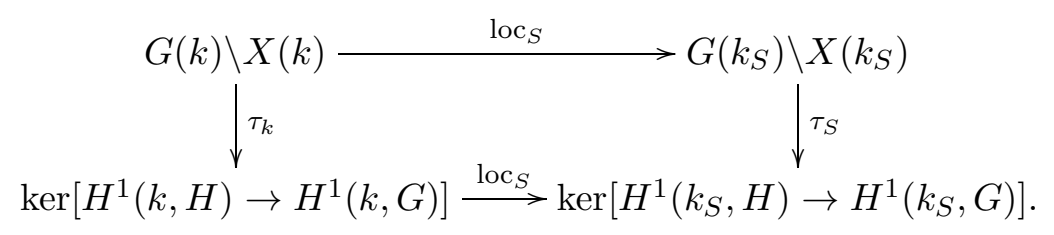

An element $x_{S} \in X\left(k_{S}\right)$ is contained in $\overline{X(k)}$ if and only if $\tau_{S}\left(G\left(k_{S}\right) \cdot x_{S}\right)$ lies in the image of the map

$$
\operatorname{ker}\left[H^{1}(k, H) \rightarrow H^{1}(k, G)\right] \stackrel{\operatorname{loc}_{S}}{\longrightarrow} \operatorname{ker}\left[H^{1}\left(k_{S}, H\right) \rightarrow H^{1}\left(k_{S}, G\right)\right] .
$$

3.3. The definition of $c_{S}$. Consider the following commutative diagram:

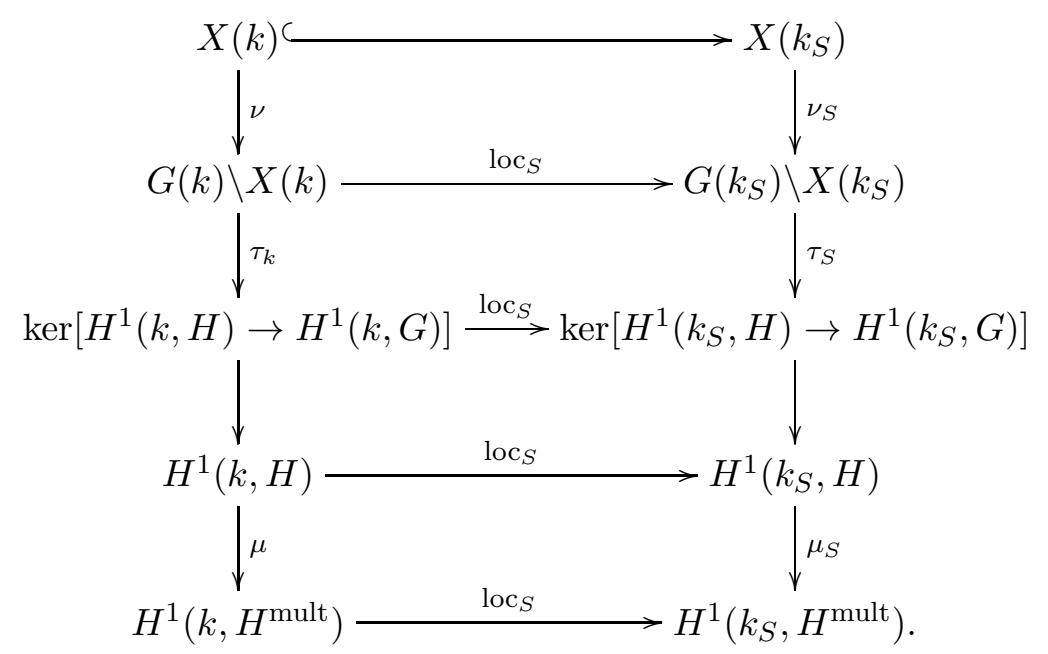

By composing the arrows in the right column of diagram (6) we obtain a map

$$
\tilde{c}_{S}: X\left(k_{S}\right) \rightarrow H^{1}\left(k_{S}, H^{\text {mult }}\right) .
$$

By composing $\tilde{c}_{S}$ with the canonical map

$$
H^{1}\left(k_{S}, H^{\text {mult }}\right) \rightarrow \mathrm{บ}_{S}^{1}\left(H^{\text {mult }}\right)
$$

we obtain a map

$$
c_{S}: X\left(k_{S}\right) \stackrel{\tilde{c}_{S}}{\longrightarrow} H^{1}\left(k_{S}, H^{\text {mult }}\right) \rightarrow \mathrm{บ}_{S}^{1}\left(H^{\text {mult }}\right) .
$$

We prove that the map $c_{S}$ is indeed an obstruction to weak approximation. 
Lemma 3.4. Let $c_{S}$ be the map defined above and let $x_{S} \in \overline{X(k)} \subset X\left(k_{S}\right)$. Then $c_{S}\left(x_{S}\right)=$ 0 .

Proof. First note that the map

$$
c_{S}: X\left(k_{S}\right) \rightarrow \mathrm{บ}_{S}^{1}\left(H^{\text {mult }}\right)
$$

is constant on $G\left(k_{S}\right)$-orbits. Since the $G\left(k_{S}\right)$-orbits are open, the map $c_{S}$ is continuous, and therefore we may assume that $x_{S} \in X(k)$. Now the assertion follows from the commutativity of diagram (6)).

Note that every step in the definition of $c_{S}$ is functorial, and therefore $c_{S}$ is functorial as well. Namely:

Lemma 3.5. Let $\phi:\left(G_{1}, H_{1}\right) \rightarrow\left(G_{2}, H_{2}\right)$ be a morphism of pairs as in 2.4 over a number field $k$ (with quasi-trivial groups $G_{1}$ and $G_{2}$ ). Set $X_{1}=G_{1} / H_{1}$ and $X_{2}=G_{2} / H_{2}$, and let $\phi_{X}: X_{1} \rightarrow X_{2}$ be the induced map. Since $\phi\left(H_{1}\right)=H_{2}$, $\phi$ induces a map $\phi^{\mu}: H_{1}^{\text {mult }} \rightarrow$ $H_{2}^{\text {mult }}$, and the following diagram commutes:

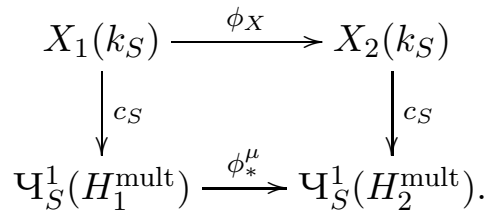

\section{Proofs: the CASE of a torus}

In this section we prove our results in the special case when our pair $(G, H)$ over $k$ is such that $G$ is a quasi-trivial torus. Note that in this case $H \subset G$ is a $k$-group of multiplicative type and $X=G / H$ has a canonical structure of a $k$-torus. We write $T=G / H$ and denote by $e$ the neutral element of $T$. Then $e \in T(k)$ is our marked point $x^{0} \in X(k)$.

Our main result in this special case is:

Theorem 4.1. Let $(G, H)$ be a pair of $k$-groups over a number field $k$ such that $G$ is a quasi-trivial k-torus. Set $T=G / H$. Then $H=H^{\text {mult }}$ is a k-group of multiplicative type, $T$ is a $k$-torus, $T\left(k_{S}\right)$ and $\overline{T(k)} \subset T\left(k_{S}\right)$ are groups, and

(i) There are canonical isomorphisms

$$
\begin{aligned}
\beta_{S} & : \amalg_{S}^{1}(\widehat{H}) \stackrel{\sim}{\rightarrow} \mathrm{Б}_{S}(T), \\
\beta_{S, \emptyset}: & \amalg_{S, \emptyset}^{1}(\widehat{H}):=\amalg_{S}^{1}(\widehat{H}) / \amalg_{\emptyset}^{1}(\widehat{H}) \stackrel{\sim}{\rightarrow} \mathrm{Б}_{S, \emptyset}(T) .
\end{aligned}
$$

(ii) We have a canonical commutative diagram

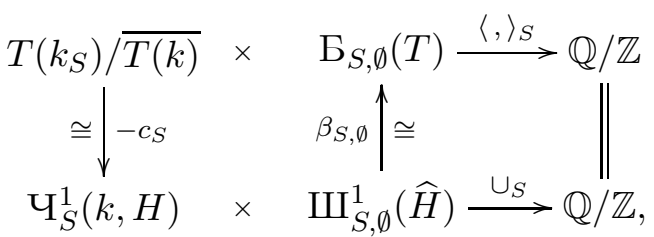

in which by abuse of notation we write $c_{S}: T\left(k_{S}\right) / \overline{T(k)} \rightarrow \mathrm{Ч}_{S}^{1}(k, H)$ for the map induced by the map $c_{S}$ defined in 9 , and by abuse of notation again we write $\langle,\rangle_{S}: T\left(k_{S}\right) / \overline{T(k)} \times$ $\mathrm{E}_{S, \emptyset}(T) \rightarrow \mathbb{Q} / \mathbb{Z}$ for the pairing induced by the Manin pairing. Moreover, in this diagram:

(i) Both vertical arrows are isomorphisms of abelian groups. 
(ii) Both pairings are perfect pairings of finite abelian groups.

When proving Theorem 4.1 we rely on Sansuc [Sa], $\S 8$. After we establish our result we shall be able to prove the following duality theorem:

Theorem 4.2. Let $H$ be a group of multiplicative type over a number field $k$. Then there is a non-degenerate pairing of finite abelian groups

$$
\amalg_{S, \emptyset}^{1}(k, \widehat{H}) \times \mathrm{\Psi}_{S}^{1}(k, H) \stackrel{\cup_{S}}{\longrightarrow} \mathbb{Q} / \mathbb{Z},
$$

where

$$
\cup_{S}=\sum_{v \in S} \operatorname{inv}_{v} \circ \cup_{k_{v}}
$$

and $\cup_{k_{v}}$ is the cup-product pairing:

$$
\cup_{k_{v}}: H^{1}\left(k_{v}, \widehat{H}\right) \times H^{1}\left(k_{v}, H\right) \stackrel{\cup}{\rightarrow} H^{2}\left(k_{v}, \mathbb{G}_{m}\right)=\operatorname{Br} k .
$$

Our first step in proving Theorem 4.1 is to describe the Brauer group of $T$ in terms of the second Galois cohomology of the Galois module $\widehat{T}$, and to describe the Manin pairing in terms of the cup product.

Lemma 4.3. Let $T$ be a torus defined over a field $\mathbb{F}$ of characteristic 0 . Then there is a canonical commutative diagram

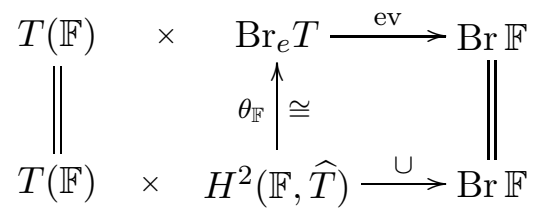

where ev is the evaluation map $(t, b) \mapsto b(t)$, and $\theta_{\mathbb{F}}$ is the canonical isomorphism of abelian groups from $\underline{\mathrm{Sa}}$, Lemma 6.9(ii). Both pairings in this diagram are additive in both arguments.

Note that the additivity of the pairing ev in the first argument means that

$$
\mathrm{ev}\left(t t^{\prime}, b\right)=\mathrm{ev}(t, b)+\mathrm{ev}\left(t^{\prime}, b\right) \quad \text { for } t, t^{\prime} \in T(\mathbb{F}), b \in \mathrm{Br}_{e} T \text {. }
$$

Proof. This is the upper half of the commutative diagram (8.11.2) in the proof of [Sa], Lemma 8.11. Note that $\theta_{\mathbb{F}}$ is the map $\theta_{\mathbb{F}}: H^{2}(\mathbb{F}, \widehat{T})=H^{2}(\mathbb{F}, U(\bar{T})) \rightarrow \operatorname{Br}_{\mathrm{a}} T=\operatorname{Br}_{e} T$ that appears in the long exact sequence in [Sa], Lemma 6.3(ii). Since the bottom pairing is additive in both arguments, so is the top one.

4.4. Now let $k$ be a number field and $S \subset \mathscr{V}(k)$ be a finite set of places. Then diagram (77) above can be used in order to compute the Manin pairing.

First we note that for every $v \in S \subset \mathscr{V}(k)$ there is a canonical inclusion $\operatorname{inv}_{v}: \operatorname{Br} k_{v} \hookrightarrow$ $\mathbb{Q} / \mathbb{Z}$. Thus for every $v \in S$ we obtain a commutative diagram

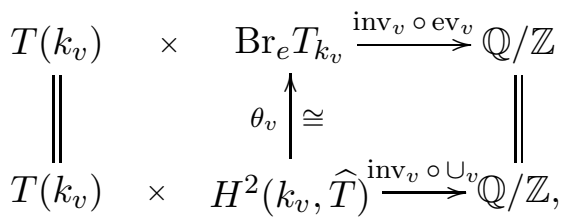

where $\theta_{v}:=\theta_{k_{v}}$. 
Now we sum several copies of diagram (8) (one for each $v \in S$ ) and obtain a commutative diagram

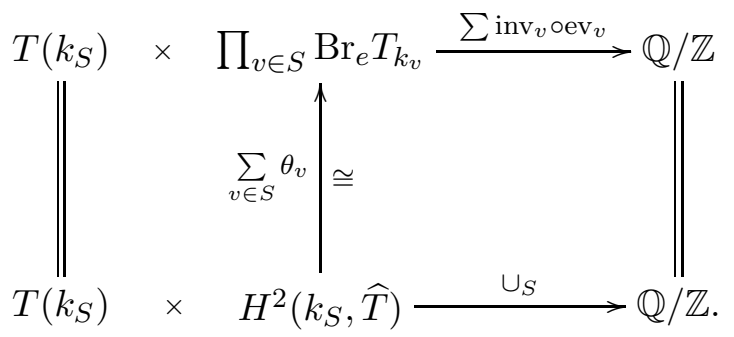

Since the isomorphism $\theta_{\mathbb{F}}$ is functorial in $\mathbb{F}$, it induces natural isomorphisms

$$
\begin{gathered}
\theta_{S}: \amalg_{S}^{2}(\widehat{T}) \stackrel{\sim}{\rightarrow} \mathrm{S}_{S}(T), \\
\theta_{S, \emptyset}: \amalg_{S, \emptyset}^{2}(\widehat{T}) \stackrel{\sim}{\rightarrow} \mathrm{Б}_{S, \emptyset}(T) .
\end{gathered}
$$

Using the natural homomorphisms

$$
\operatorname{loc}_{S}: \amalg_{S}^{2}(\widehat{T}) \rightarrow H^{2}\left(k_{S}, \widehat{T}\right), \quad \mathrm{Б}_{S}(T) \rightarrow \prod_{v \in S} \mathrm{Br}_{e} T_{k_{v}},
$$

we obtain from diagram (9) a commutative diagram

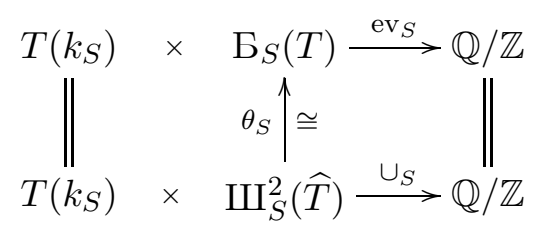

where $\theta_{S}$ is isomorphism (10) and

$$
\operatorname{ev}_{S}\left(t_{S}, b\right)=\sum_{v \in S} \operatorname{inv}_{v}\left(b\left(t_{v}\right)\right) \text { for } b \in \mathrm{Б}_{S}(T) \subset \operatorname{Br}_{e} T \subset \operatorname{Br}_{1} T \text { and } t_{S} \in T\left(k_{S}\right) .
$$

But $v_{S}$ is exactly the Manin pairing (10) (denoted by $\langle,\rangle_{S}$ there). Thus we obtain the following commutative diagram, containing the map induced by the Manin pairing as the top pairing:

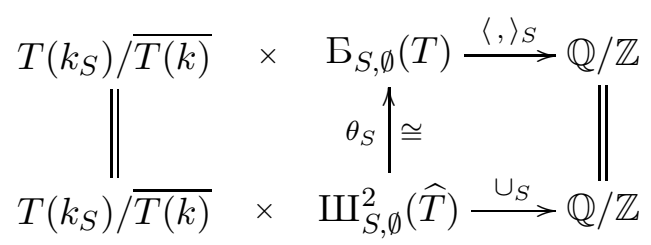

Note that we may write $T\left(k_{S}\right) / \overline{T(k)}$ instead of $T\left(k_{S}\right)$, because we know that the Manin pairing vanishes on $\overline{T(k)}$ and is additive in the first argument.

The next step in our proof will be using connecting maps in order to reduce the second Galois cohomology that appears in diagram (12) to the first Galois cohomology.

Lemma 4.5. Let $1 \rightarrow H \rightarrow G \rightarrow T \rightarrow 1$ be a short exact sequence of groups of multiplicative type over a field $\mathbb{F}$ of characteristic 0 , and let $0 \rightarrow \widehat{T} \rightarrow \widehat{G} \rightarrow \widehat{H} \rightarrow 0$ be the dual 
exact sequence. Then the following diagram anti-commutes:

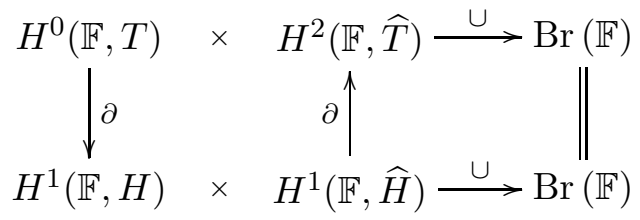

Proof. The proof is similar to that of $\left[\mathrm{Sa}\right.$, Lemma 8.11. Let $t \in T(\mathbb{F})$ and $f \in H^{1}(\mathbb{F}, \widehat{H})$. Let us lift $t$ and $f$ to $t_{G} \in C^{0}(\mathbb{F}, G)$ and $f_{G} \in C^{1}(\mathbb{F}, \widehat{G})$, respectively. We have $d\left(t_{G} \cup f_{G}\right)=$ $d\left(t_{G}\right) \cup f_{G}+t_{G} \cup d\left(f_{G}\right)$. By passing to cohomology classes we obtain $\partial t \cup f=-t \cup \partial f$.

4.6. By taking the anticommutative diagram (13) with $\mathbb{F}=k$ and $\mathbb{F}=k_{v}, v \in S$, and arguing as in 4.4, we obtain an anticommutative diagram

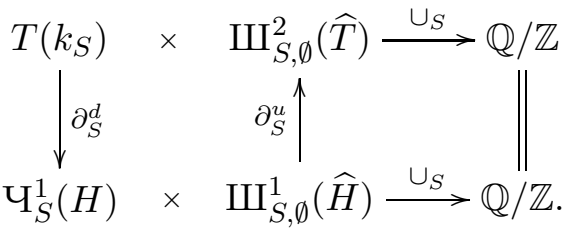

Note that in diagram (14) we may write $\mathrm{\Psi}_{S}^{1}(H)$ instead of $H^{1}\left(k_{S}, H\right)$, because by the short exact sequence

the image of the map

$$
0 \rightarrow \mathrm{Br} k \rightarrow \oplus \operatorname{Br} k_{v} \stackrel{\sum \operatorname{inv}_{v}}{\longrightarrow} \mathbb{Q} / \mathbb{Z}
$$

lies in the left kernel of the pairing

$$
\operatorname{loc}_{S}: H^{1}(k, H) \rightarrow H^{1}\left(k_{S}, H\right)
$$

$$
H^{1}\left(k_{S}, H\right) \times \amalg_{S}^{1}(\widehat{H}) \stackrel{\cup_{S}}{\longrightarrow} \mathbb{Q} / \mathbb{Z} .
$$

Now we prove that the map $\partial_{S}^{d}$ from diagram (14) is exactly our obstruction $c_{S}$ and that it induces an isomorphism $T\left(k_{S}\right) / \overline{T(k)} \rightarrow \mathrm{บ}_{S}^{1}(H)$.

Lemma 4.7. Let a pair of $k$-groups $(G, H)$ be such that $G$ is a (quasi-trivial) torus. Set $T=G / H$. We denote by $\alpha: H^{1}\left(k_{S}, H\right) \rightarrow \mathrm{Ч}_{S}^{1}(H)$ the canonical epimorphism, and by $\partial_{S}$ the map

$$
T\left(k_{S}\right) \stackrel{\partial_{S}}{\longrightarrow} H^{1}\left(k_{S}, H\right),
$$

obtained from the short exact sequence of groups of multiplicative type $1 \rightarrow H \rightarrow G \rightarrow$ $T \rightarrow 1$. Then

(i) $\alpha \circ \partial_{S}=c_{S}$;

(ii) $c_{S}$ is a homomorphism;

(iii) $c_{S}$ is surjective;

(iv) $\operatorname{ker} c_{S}=\overline{T(k)}$.

Proof. From the short exact sequence of groups of multiplicative type

$$
1 \rightarrow H \rightarrow G \stackrel{\rho}{\rightarrow} T \rightarrow 0
$$

we obtain a commutative diagram of abelian groups

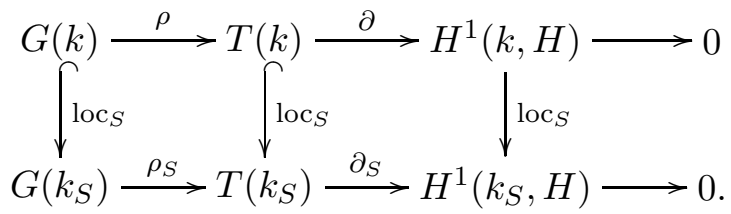


Since $H^{\text {mult }}=H$, we have $c_{S}=\alpha \circ \tau_{S} \circ \nu_{S}$ (see $\sqrt{3.3}$ ), and (ii) follows from the equality $\partial_{S}=\tau_{S} \circ \nu_{S}$. Since $\alpha$ and $\partial_{S}$ are both homomorphisms, we see that $c_{S}$ is a homomorphism, which proves (iii). Assertion (iii) follows from the surjectivity of $\alpha$ and $\partial_{S}$.

We prove (iv) by diagram chasing in diagram (15). Since we know that $\overline{T(k)} \subseteq \operatorname{ker} c_{S}$, it suffices to show that $\operatorname{ker} c_{S} \subseteq \overline{T(k)}$. Let $t_{S} \in \operatorname{ker} c_{S} \subset T\left(k_{S}\right)$, i.e. $\alpha\left(\partial_{S}\left(t_{S}\right)\right)=0$. Then there exists $h \in H^{1}(k, H)$ such that $\partial_{S}\left(t_{S}\right)=\operatorname{loc}_{S}(h)$. We can find $t \in T(k)$ such that $\partial(t)=h$ (because $\partial$ is surjective). We have

$$
\partial_{S}\left(t_{S}\right)=\operatorname{loc}_{S}(h)=\operatorname{loc}_{S}(\partial(t))=\partial_{S}\left(\operatorname{loc}_{S}(t)\right),
$$

and therefore $t_{S}-t \in \rho_{S}\left(G\left(k_{S}\right)\right)$. Thus we have showed that ker $c_{S} \subseteq \rho_{S}\left(G\left(k_{S}\right)\right)+T(k)$, and it suffices to prove that $\rho_{S}\left(G\left(k_{S}\right)\right)+T(k) \subseteq \overline{T(k)}$. Since $G$ is quasi-trivial, it has the weak approximation property (cf. [CT], Proposition 9.2), and therefore $\overline{G(k)}=G\left(k_{S}\right)$. We obtain:

$$
\rho_{S}\left(G\left(k_{S}\right)\right)+T(k) \subseteq \overline{\rho_{S}(G(k))}+T(k) \subseteq \overline{\rho(G(k))+T(k)}=\overline{T(k)} .
$$

The following lemma has been widely used, see e.g. [B1], Proof of Lemma 4.4, or [B2, $\S 3.5$, but we do not know a reference where it was stated, so we state and prove it here.

Lemma 4.8. Consider a commutative diagram of abelian groups with exact rows

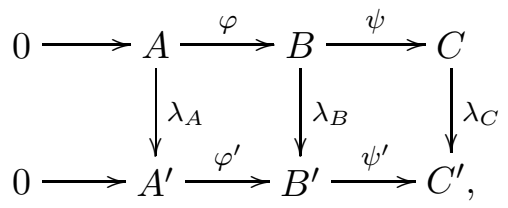

then the induced sequence

$$
0 \rightarrow \operatorname{ker} \lambda_{A} \stackrel{\varphi_{*}}{\longrightarrow} \operatorname{ker} \lambda_{B} \stackrel{\psi_{*}}{\longrightarrow} \operatorname{ker} \lambda_{C}
$$

is exact.

Proof. We replace $C$ and $C^{\prime}$ by $\operatorname{im} \psi$ and $\operatorname{im} \psi^{\prime}$, resp., and apply the Snake Lemma.

We proceed by proving that the map $\partial_{S}^{u}$ from diagram (14) is an isomorphism.

Lemma 4.9. Let $1 \rightarrow H \rightarrow G \rightarrow T \rightarrow 1$ be a short exact sequence of groups of multiplicative type such that $G$ is a quasi-trivial torus. Let

$$
\partial: \amalg_{S}^{1}(\widehat{H}) \rightarrow \amalg_{S}^{2}(\widehat{T})
$$

be the map induced by the connecting map obtained from the short exact sequence

$$
0 \rightarrow \widehat{T} \rightarrow \widehat{G} \rightarrow \widehat{H} \rightarrow 0 .
$$

Then $\partial$ is an isomorphism.

Proof. From the short exact sequence $0 \rightarrow \widehat{T} \stackrel{i}{\rightarrow} \widehat{G} \rightarrow \widehat{H} \rightarrow 0$ we obtain a commutative diagram with exact rows

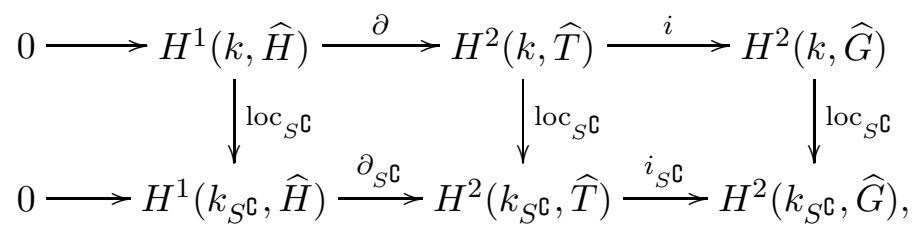


where $S^{\complement}=\mathscr{V}(k) \backslash S$. Since the $k$-torus $G$ is quasi-trivial, by [Sa], (1.9.1), we have $\amalg_{S}^{2}(\widehat{G})=0$. Now by Lemma 4.8 the induced homomorphism (16) is an isomorphism.

Using Lemmas 4.7 and 4.9, we can rewrite diagram (14).

Lemma 4.10. Let $(G, H)$ be a pair of k-groups such that $G$ is a quasi-trivial torus. Set $T=G / H$. Then we have a commutative diagram

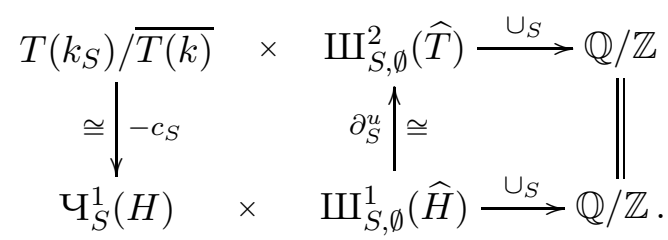

In this diagram:

(i) Both vertical arrows are isomorphisms.

(ii) Both pairings are perfect pairings of finite abelian groups.

Proof. First note that we can write in diagram (14) $T\left(k_{S}\right) / \overline{T(k)}$ instead of $T\left(k_{S}\right)$, because $\overline{T(k)}$ lies in the left kernel of the top pairing by diagram (12), and in the kernel of $\partial_{S}^{d}=c_{S}$ by Lemma 4.7. By Lemmas 4.7 and 4.9, diagram (17) is just a version of diagram (14), where we take $c_{S}$ with the negative sign in order to obtain a commutative diagram from the anticommutative diagram (14). Also (i) follows from Lemmas 4.7 and 4.9 ,

It remains to prove (ii). By Ono's lemma (cf. [O], Theorem 1.5.1) there is an exact sequence

$$
1 \rightarrow B \rightarrow Q_{1} \rightarrow T^{m} \times Q_{2} \rightarrow 1
$$

such that $m>0$ is an integer, $Q_{1}$ and $Q_{2}$ are quasi-trivial $k$-tori and $B$ is a finite abelian $k$-group.

Now we construct diagram (17) for the pair of $k$-groups $G=Q_{1}$ and $H=B$, then $Q_{1} / B=T^{m} \times Q_{2}$. Using (ii) we obtain

$$
\begin{array}{ccc}
\left(T\left(k_{S}\right) / \overline{T(k)}\right)^{m} \oplus Q_{2}\left(k_{S}\right) / \overline{Q_{2}(k)} & \times\left(\amalg_{S, \emptyset}^{2}(\widehat{T})\right)^{m} \oplus \amalg_{S, \emptyset}^{2}\left(\widehat{Q_{2}}\right) \stackrel{\cup_{S}}{\longrightarrow} \mathbb{Q} / \mathbb{Z} \\
\cong \mid-c_{S} & \partial_{S}^{u} \mid \cong \\
\mathrm{บ}_{S}^{1}(B) & \times & \amalg_{S, \emptyset}^{1}(\widehat{B}) \stackrel{\cup_{S}}{\longrightarrow} \mathbb{Q} / \mathbb{Z} .
\end{array}
$$

Since $Q_{2}$ is a quasi-trivial torus, it has the weak approximation property,

i.e. $Q_{2}\left(k_{S}\right) / \overline{Q_{2}(k)}=0$. Further, by $\left[\underline{\mathrm{Sa}},(1.9 .1)\right.$, we have $\amalg_{S}^{2}\left(\widehat{Q_{2}}\right)=0$. Thus we have a commutative diagram

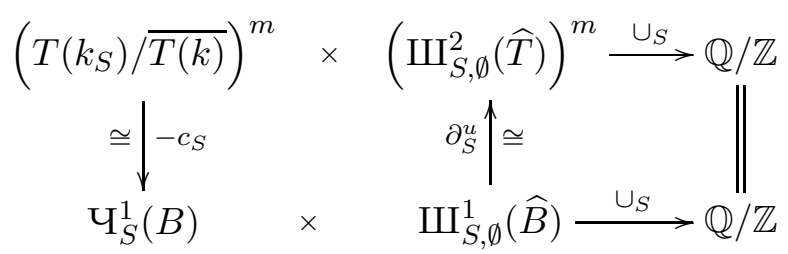

where the vertical arrows are isomorphisms.

But by $[\mathrm{Sa}$, the proof of Lemma 1.4, the bottom pairing in diagram (18) is a perfect pairing of finite abelian groups. Therefore the top pairing:

$$
\left(T\left(k_{S}\right) / \overline{T(k)}\right)^{m} \times\left(\amalg_{S, \emptyset}^{2}(\widehat{T})\right)^{m} \stackrel{\cup_{S}}{\longrightarrow} \mathbb{Q} / \mathbb{Z}
$$


is a perfect pairing of finite abelian groups, and clearly the same is true for the pairing

$$
T\left(k_{S}\right) / \overline{T(k)} \times \amalg_{S, \emptyset}^{2}(\widehat{T}) \stackrel{\cup_{S}}{\longrightarrow} \mathbb{Q} / \mathbb{Z} .
$$

Now (ii) gives us (iii).

Now we complete the proof of Theorem 4.1 .

Proof of Theorem 4.1. By composing the isomorphisms:

$$
\amalg_{S}^{1}(\widehat{H}) \stackrel{\sim}{\rightarrow} \amalg_{S}^{2}(\widehat{T}) \stackrel{\sim}{\rightarrow} \mathrm{Б}_{S}(T)
$$

from (16) and (10), respectively, we obtain isomorphisms

$$
\begin{aligned}
\beta_{S} & : \amalg_{S}^{1}(\widehat{H}) \stackrel{\sim}{\rightarrow} \mathrm{Б}_{S}(T), \\
\beta_{S, \emptyset}: & \amalg_{S, \emptyset}^{1}(\widehat{H}):=\amalg_{S}^{1}(\widehat{H}) / \amalg_{\emptyset}^{1}(\widehat{H}) \stackrel{\sim}{\rightarrow} \mathrm{Б}_{S, \emptyset}(T),
\end{aligned}
$$

so we have proved (i). We prove (ii) $(a, b)$. First we put diagram (12) on top of diagram (17) and obtain the following commutative diagram, in which the vertical arrows are isomorphisms:

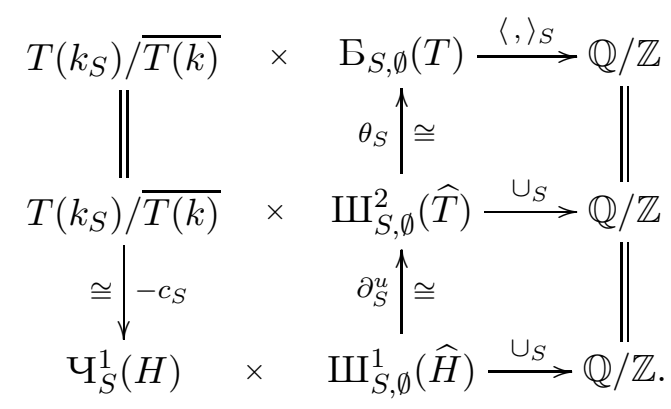

By Lemma 4.10)(ii) the pairings in the two lower rows of diagram (19) are perfect pairings of finite abelian groups. Since all vertical arrows of diagram (19) are isomorphisms, we see that the pairing in the top row is a perfect pairing of finite abelian groups as well. We conclude that the diagram

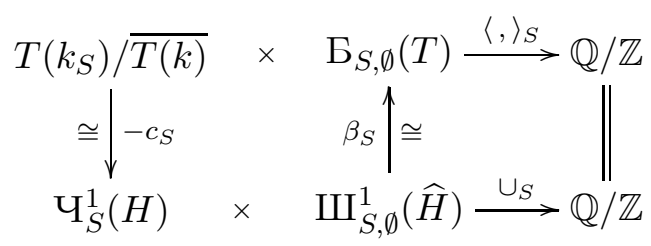

satisfies (a,b), as required.

Proof of Theorem 4.2. Let $H$ be a $k$-group of multiplicative type. Since the Galois module $\widehat{H}$ is a quotient of a permutation Galois module, there exists an embedding $H \hookrightarrow G$ into a quasi-trivial $k$-torus $G$. By applying Lemma 4.10 to the pair $(G, H)$ we obtain the desired duality.

\section{Proofs: the General CASE}

In this section we prove our results in the general case by using morphisms of pairs $(G, H)$ which preserve both the Brauer-Manin obstruction and our cohomological obstruction $c_{S}$. Using such morphisms we reduce the proof of Theorem 0.2 to the case when $G$ is a torus, which was dealt with in the previous section.

The following theorem is the main result of this paper. 
Theorem 5.1. Let $(G, H)$ be a pair of k-groups as in 2.4 (with quasi-trivial $G$ ) over a number field $k$, and let $S \subset \mathscr{V}(k)$ a finite set of places of $k$. Then

(i) There are canonical isomorphisms

$$
\begin{gathered}
\beta_{S}: \amalg_{S}^{1}(\widehat{H}) \stackrel{\sim}{\rightarrow} \mathrm{Б}_{S}(X), \\
\beta_{S, \emptyset}: \amalg_{S, \emptyset}^{1}(\widehat{H}) \stackrel{\sim}{\rightarrow} \mathrm{Б}_{S, \emptyset}(X) .
\end{gathered}
$$

(ii) The following canonical diagram commutes:

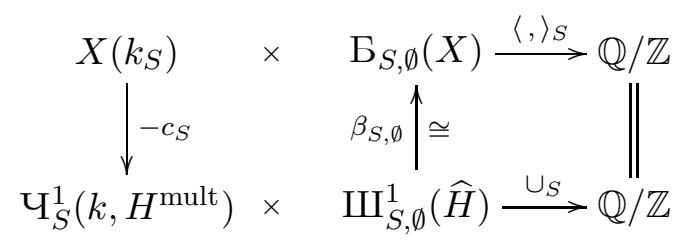

In diagram (20) the bottom pairing is a perfect pairing of finite groups.

In order to prove Theorem 5.1 we shall construct auxiliary pairs of $k$-groups and morphisms of pairs, similar to [B1].

5.2. Let $(G, H)$ be a pair of $k$-groups as in 2.4 (with quasi-trivial $G$ ) over a number field $k$. Choose an embedding $i: H^{\text {mult }} \rightarrow Q$ into a quasi-trivial torus $Q$. Consider the embedding

$$
i_{*}: H \rightarrow G \times{ }_{k} Q, \quad h \mapsto(h, i(\mu(h))),
$$

where $\mu: H \rightarrow H^{\text {mult }}$ is the canonical epimorphism. Set $G_{Y}=G \times{ }_{k} Q, H_{Y}=i_{*}(H)$. The pair $\left(G_{Y}, H_{Y}\right)$ defines a homogeneous space $Y=G_{Y} / H_{Y}=\left(G \times_{k} Q\right) / i_{*}(H)$ with a marked point $y^{0}$. The projection map $\pi: G_{Y}=G \times Q \rightarrow G$ is surjective and satisfies $\pi\left(H_{Y}\right)=H$, and therefore it defines a morphism of pairs as in $2.4 \pi:\left(G_{Y}, H_{Y}\right) \rightarrow(G, H)$, which in turn defines a morphism of varieties with marked points $\pi_{*}:\left(Y, y^{0}\right) \rightarrow\left(X, x^{0}\right)$. Note that the map $H_{Y}^{\text {mult }} \rightarrow G_{Y}^{\text {tor }}$ is injective and that $\left(Y, \pi_{*}\right)$ is a torsor over $X$ under $Q$. Since $Q$ is a quasi-trivial torus, we see that for any field $\mathbb{F}$ containing $k$ the map $\pi_{*}: Y(\mathbb{F}) \rightarrow X(\mathbb{F})$ is surjective.

Lemma 5.3. With notation and assumptions of 5.2 we have:

(i) $U(\bar{Y}) \cong G_{Y}^{\text {tor }} / H_{Y}^{\text {mult }}$.

(ii) $\operatorname{Pic} \bar{Y}=0$.

(iii) There is a canonical functorial isomorphism

$$
\theta: H^{2}(k, U(\bar{Y})) \stackrel{\sim}{\rightarrow} \operatorname{Br}_{\mathrm{a}} Y \stackrel{\sim}{\rightarrow} \operatorname{Br}_{y^{0}} Y
$$

where $\bar{Y}=Y \times_{k} \bar{k}$.

Proof. Since the map $H_{Y}^{\text {mult }} \rightarrow G_{Y}^{\text {tor }}$ is injective, (ii) and (iii) follow from Proposition 2.5. It remains to prove (iii). The Hochschild-Serre spectral sequence

$$
H^{p}\left(\operatorname{Gal}(\bar{k} / k), H^{q}\left(\bar{Y}, \mathbb{G}_{m}\right)\right) \Rightarrow H^{n}\left(Y, \mathbb{G}_{m}\right)
$$

gives rise to an exact sequence

$$
H^{0}(k, \operatorname{Pic} \bar{Y}) \rightarrow H^{2}(k, U(\bar{Y})) \stackrel{\theta_{\mathrm{a}}}{\rightarrow} \operatorname{Br}_{\mathrm{a}} Y \rightarrow H^{1}(k, \operatorname{Pic} \bar{Y})
$$

(see [Sa], Lemma 6.3(ii)). We see from (iii) that $\theta_{\mathrm{a}}$ is an isomorphism. Composing $\theta_{\mathrm{a}}$ with the canonical isomorphism $\operatorname{Br}_{\mathrm{a}} Y \stackrel{\sim}{\rightarrow} \mathrm{Br}_{y^{0}} Y$, we obtain the desired isomorphism $\theta$. 
5.4. Consider the canonical epimorphism $\mu: G_{Y} \rightarrow G_{Y}^{\text {mult }}=G_{Y}^{\text {tor }}$. Then the following diagram commutes and has injective horizontal arrows and surjective vertical arrows:

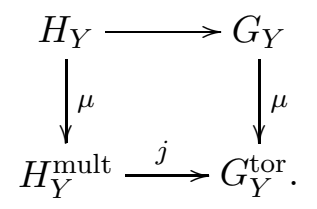

We construct a new pair $\left(G_{Z}, H_{Z}\right)$ as follows: $G_{Z}=G_{Y}^{\text {tor }}, H_{Z}=j\left(H_{Y}^{\text {mult }}\right)$. Set $Z=$ $G_{Z} / H_{Z}$, it is a $k$-torus, we denote by $z^{0}$ its identity element. We have a morphism of pairs $\mu:\left(G_{Y}, H_{Y}\right) \rightarrow\left(G_{Z}, H_{Z}\right)$ as in 2.4 and the induced morphism of homogeneous spaces $\mu_{*}:\left(Y, y^{0}\right) \rightarrow\left(Z, z^{0}\right)$. Thus we have diagrams
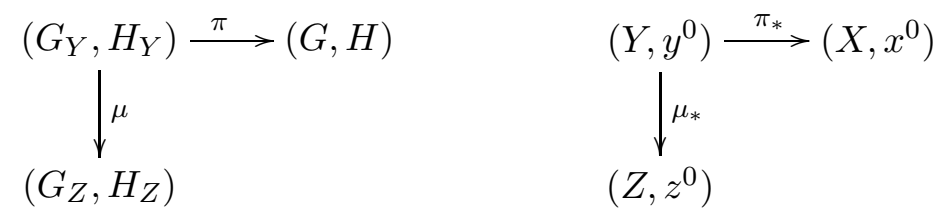

Since $G_{Z}$ is a torus, we know that Theorem 5.1 is true for $Z$ (this is Theorem 4.1). So all we have to do is to prove that both $\mu$ and $\pi$ preserve ob $b_{S}$ and $c_{S}$.

Lemma 5.5. The following diagrams commute and all the vertical arrows marked with $(\cong)$ are isomorphisms.
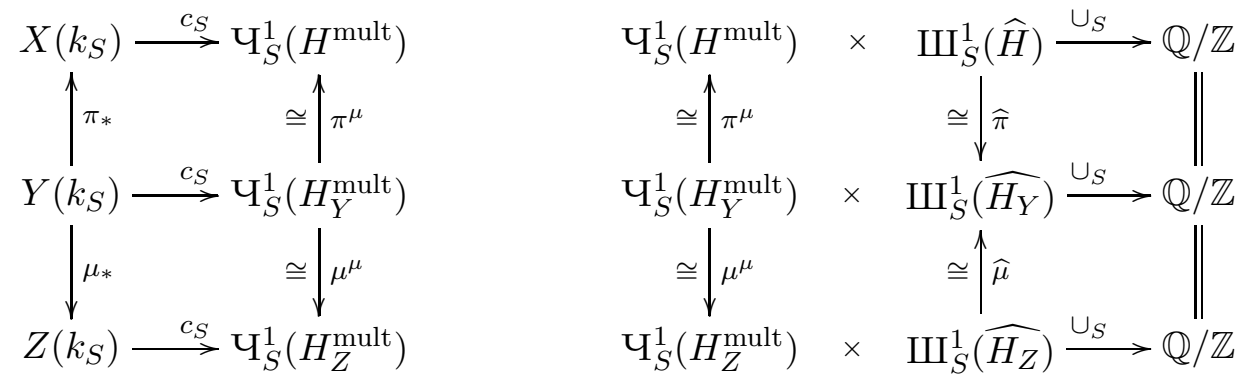

Proof. The lemma follows from the functoriality of $c_{S}$ and from the fact that $\pi^{\mu}: H_{Y}^{\text {mult }} \rightarrow$ $H^{\text {mult }}$ and $\mu^{\mu}: H_{Y}^{\text {mult }} \rightarrow H_{Z}^{\text {mult }}$ are isomorphisms.

Lemma 5.6. The following diagram commutes and all the vertical arrows marked with $(\cong)$ are isomorphisms.

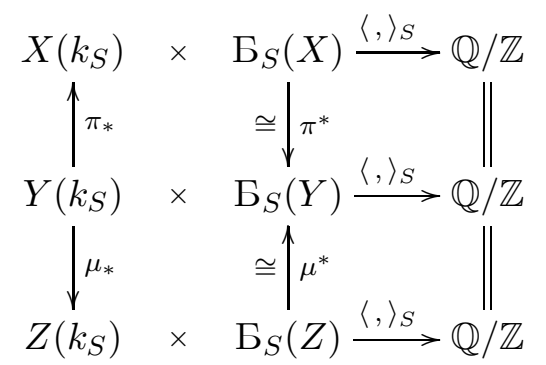

Proof. The commutativity of the diagram follows from the functoriality of $\langle,\rangle_{S}$. We prove that $\pi^{*}$ is an isomorphism. Since $Y$ is a torsor over $X$ under $Q$, by [Sa], (6.10.3), there is an exact sequence

$$
\operatorname{Pic} Q \rightarrow \operatorname{Br}_{1} X \stackrel{\pi^{*}}{\rightarrow} \operatorname{Br}_{1} Y \rightarrow \operatorname{Br}_{e} Q
$$


hence an exact sequence

$$
\operatorname{Pic} Q \rightarrow \operatorname{Br}_{x^{0}} X \stackrel{\pi^{*}}{\rightarrow} \operatorname{Br}_{y^{0}} Y \rightarrow \operatorname{Br}_{e} Q \text {. }
$$

By [Sa], Lemma 6.9(ii), we have $\operatorname{Pic} Q \cong H^{1}(k, \widehat{Q})=0$ (because $Q$ is quasi-trivial) and $\operatorname{Br}_{e} Q \cong H^{2}(k, \widehat{Q})$. We obtain a commutative diagram

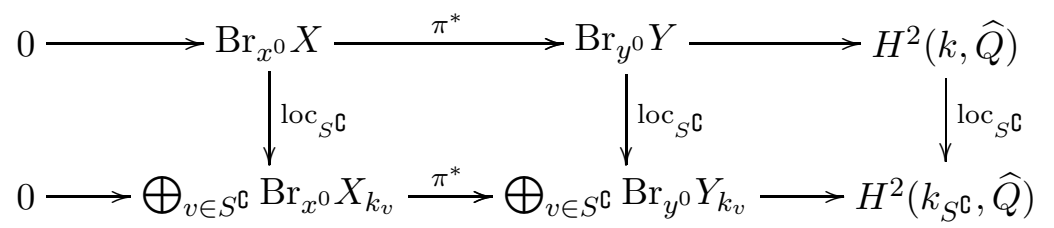

where $S^{\complement}=\mathscr{V}(k) \backslash S$. By [Sa], (1.9.1), we have $\amalg_{S}^{2}(\widehat{Q})=0$. By Lemma 4.8 our diagram (24) induces an isomorphism

$$
\pi^{*}: \mathrm{Б}_{S}(X) \rightarrow \mathrm{Б}_{S}(Y) .
$$

It is remains to prove that $\mu^{*}$ is an isomorphism. From the construction of $\left(G_{Z}, H_{Z}\right)$ we obtain a commutative diagram with injective horizontal arrows and bijective vertical arrows

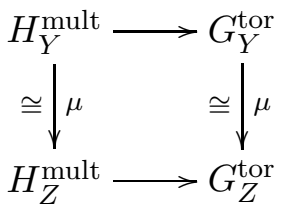

From this diagram we obtain an isomorphism $\mu_{*}: G_{Y}^{\text {tor }} / H_{Y}^{\text {mult }} \rightarrow G_{Z}^{\text {tor }} / H_{Z}^{\text {mult }}$. By Lemma 5.3 and Diagram (8) in $§ 4.4$ we have canonical isomorphisms

$$
\begin{aligned}
& \operatorname{Br}_{z^{0}} Z \cong H^{2}(k, U(\bar{Z})) \cong H^{2}\left(k, G_{Z}^{\text {tor }} / H_{Z}^{\text {mult }}\right), \\
& \operatorname{Br}_{y^{0}} Y \cong H^{2}(k, U(\bar{Y})) \cong H^{2}\left(k, G_{Y}^{\text {tor }} / H_{Y}^{\text {mult }}\right) \text {. }
\end{aligned}
$$

We obtain a commutative diagram

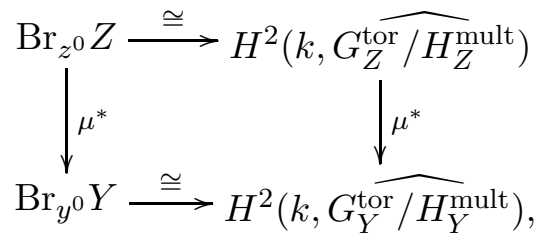

where the right-hand vertical arrow is an isomorphism. It follows that the left-hand vertical arrow in this diagram is an isomorphism, hence the homomorphism $\mu^{*}: \mathrm{Б}_{S}(Z) \rightarrow \mathrm{Б}_{S}(Y)$ is an isomorphism.

Now we complete the proof of Theorem 5.1 ,

Proof of Theorem 5.1. We prove (ii). Since $G_{Z}$ is a quasi-trivial torus, by Theorem 4.1(1) we have an isomorphism

$$
\beta_{S}^{Z}: \amalg_{S}^{1}\left(\widehat{H_{Z}}\right) \stackrel{\sim}{\rightarrow} \mathrm{5}_{S}(Z)
$$


Using diagrams (22) and (23), we obtain a diagram with bijective arrows

$$
\begin{array}{r}
\amalg_{S}^{1}(\widehat{H}) \stackrel{\widehat{\pi}}{\cong} \amalg_{S}^{1}\left(\widehat{H_{Y}}\right) \stackrel{\widehat{\mu}}{\cong} \amalg_{S}^{1}\left(\widehat{H_{Z}}\right) \\
\cong \mid \beta_{S}^{Z} \\
\mathrm{~L}_{S}(X) \stackrel{\pi^{*}}{\cong} \mathrm{D}_{S}(Y) \stackrel{\mu^{*}}{\cong} \mathrm{D}_{S}(Z)
\end{array}
$$

This diagram gives us the required isomorphism

$$
\beta_{S}: \amalg_{S}^{1}(\widehat{H}) \stackrel{\sim}{\rightarrow} \mathrm{Б}_{S}(X) .
$$

The map $\beta_{S}$ induces an isomorphism

$$
\beta_{S, \emptyset}: \amalg_{S, \emptyset}^{1}(\widehat{H}) \stackrel{\sim}{\rightarrow} \mathrm{Б}_{S, \emptyset}(X) .
$$

We prove (iii). By Theorem 4.2 the bottom pairing in diagram (20) is a perfect pairing of finite groups. We prove the commutativity of this diagram by a diagram chase in diagrams (22) and (22).

Let $x_{S} \in X\left(k_{S}\right)$ and $t \in \amalg_{S}^{1}(\widehat{H})$. Since $Y \rightarrow X$ is a torsor under the quasi-trivial torus $Q$, there exists $y_{S} \in Y\left(k_{S}\right)$ such that $\pi\left(y_{S}\right)=x_{S}$. We set $z_{S}=\mu\left(y_{S}\right) \in Z\left(k_{S}\right)$. We set $t_{Y}:=\widehat{\pi}(t) \in \amalg_{S}^{1}\left(\widehat{H_{Y}}\right)$ and $t_{Z}=\widehat{\mu}^{-1}\left(t_{Y}\right) \in \amalg_{S}^{1}\left(\widehat{H_{Z}}\right)$. By diagrams (22) we have

$$
\cup_{S}\left(-c_{S}\left(x_{S}\right), t\right)=\cup_{S}\left(-c_{S}\left(y_{S}\right), t_{Y}\right)=\cup_{S}\left(-c_{S}\left(z_{S}\right), t_{Z}\right) .
$$

By Theorem 4.1 we have

$$
\cup_{S}\left(-c_{S}\left(z_{S}\right), t_{Z}\right)=\left\langle z_{S}, \beta_{S}^{Z}\left(t_{Z}\right)\right\rangle_{S}
$$

Set $b_{Z}=\beta_{S}^{Z}\left(t_{Z}\right)$, then

$$
\cup_{S}\left(-c_{S}\left(z_{S}\right), t_{Z}\right)=\left\langle z_{S}, b_{Z}\right\rangle_{S}
$$

Set $b_{Y}=\mu^{*}\left(b_{Z}\right) \in \mathrm{Б}_{S}(Y), \quad b_{X}=\left(\pi^{*}\right)^{-1}\left(b_{Y}\right) \in \mathrm{Б}_{S}(X)$, then by diagram (23) we have

$$
\left\langle z_{S}, b_{Z}\right\rangle_{S}=\left\langle y_{S}, b_{Y}\right\rangle_{S}=\left\langle x_{S}, b_{X}\right\rangle_{S} \text {. }
$$

Since $b_{X}=\beta_{S}(t)$, we obtain that

$$
\cup_{S}\left(-c_{S}\left(x_{S}\right), t\right)=\left\langle x_{S}, \beta_{S}(t)\right\rangle_{S},
$$

as required.

\section{REFERENCES}

[B1] M. Borovoi, The Brauer-Manin obstruction for homogeneous spaces with connected or abelian stabilizer, J. reine angew. Math. 473 (1996), 181-194.

[B2] M. Borovoi, A cohomological obstruction to the Hasse principle for homogeneous spaces, Math. Ann. 314 (1999), 491-504.

[B3] M. Borovoi, The defect of weak approximation for homogeneous spaces, Ann. Fac. Sci. Toulouse 8 (1999), 219-233.

[BD] M. Borovoi and C. Demarche, Manin obstruction to strong approximation for homogeneous spaces, To appear in Comment. Math. Helv., arXiv:0912.0408v2 [math.NT]

[CT] J.-L. Colliot-Thélène, Résolutions flasques des groupes linéaires connexes, J. reine angew. Math. 618 (2008), 77-133.

[CTS] J.-L. Colliot-Thélène et J.-J. Sansuc, La descente sur une variété rationnelle définie sur un corps de nombres, C. R. A. S. Paris 284 (1977), A1215-1218.

[D1] C. Demarche, Groupe de Brauer non ramifié d'espaces homogènes à stabilisateurs finis, Math. Ann. 346 (2010), 949-968.

[D2] C. Demarche, Suites de Poitou-Tate pour les complexes de tores à deux termes, Int. Math. Res. Notices 2011, 135-174.

$[\mathrm{H}] \quad$ D. Harari, Quelques propriétés d'approximation reliés à la cohomologie galoisienne d'un groupe algébrique fini, Bull. Soc. Math. France 135 (2007), 549-564. 
[M] Yu.I. Manin, Le groupe de Brauer-Grothendieck en géométrie diophantienne, in: Actes du Congrès Intern. Math. (Nice, 1970), Tome 1, Gauthier-Villars, Paris, 1971, pp. 401-411.

[O] T. Ono, Arithmetic of algebraic tori, Annals of Math. 74 (1961), 101-139.

[P] V.L. Popov, Picard groups of homogeneous spaces of linear algebraic groups and one dimensional homogeneous vector bundles. Math. USSR Izv. 8 (1974) 301-327.

[R] M. Rosenlicht, Toroidal algebraic groups, Proc. AMS 12 (1961), 984-988.

[Sa] J.-J. Sansuc, Groupe de Brauer et arithmétique des groupes algébriques linéaires sur un corps de nombres, J. reine angew. Math. 327 (1981), 12-80.

[Se] J.-P. Serre, Cohomologie galoisienne, Lecture Notes in Math. 5, 5ème ed., Springer-Verlag, Berlin 1994.

[Sk] A.N. Skorobogatov, Torsors and Rational Points, Cambridge Tracts in Mathematics 144, Cambridge University Press, Cambridge 2001.

Borovoi: Raymond and Beverly Sackler School of Mathematical Sciences, Tel Aviv UniVERsity, Tel Aviv 69978, IsRAel

E-mail address: borovoi@post.tau.ac.il

Schlank: Institute of Mathematics, Hebrew University, Giv'at-Ram, Jerusalem 91904, ISRAEL

E-mail address: tomer.schlank@gmail.com 